\title{
A General Statistical Channel Model for Mobile Satellite Systems
}

\author{
Yongjun Xie and Yuguang Fang, Senior Member, IEEE
}

\begin{abstract}
In order to guarantee the service quality and reliability for mobile satellite communication systems, we have to take into account outages due to obstruction of the line-of-sight path between a satellite and a mobile terminal as well as the signal fluctuation caused by interference from multipath radio waves. Thus, we need a good characterization for the satellite propagation channel. In this paper, we derive a general statistical model for mobile communication systems based on propagation scattering theory. Both the probability density function (pdf) of the received signal envelope and that of received signal power are given. This model is shown to be more general than previously known statistical models and can provide a better fit to the experimental data. This new model will play a significant role in the design of mobile satellite communication systems.
\end{abstract}

Index Terms-Channel modeling, fading, mobile satellite communications, propagation, statistical models.

\section{INTRODUCTION}

I T IS COMMONLY accepted that satellite communications systems [in particular, the low earth orbit (LEO) systems] are the de facto solution for providing the real personal communications services (PCS's) to the users either stationary or on the move anywhere, anytime and in any format (voice, data, and multimedia) [1].

Satellite communication systems have provided international telecommunications services since the 1960's. These systems were augmented in the 1970's and 1980's with regional satellite systems, national systems, and private network-based very small aperture terminals (VSAT's). Throughout this period, systems have been based exclusively on satellites in geosynchronous orbit communicating with earth stations using high gain fixed antennas. As the systems have evolved, the original 30-m-diameter Intelsat earth stations have evolved into 1.2 -m $K u$-band VSAT's for business and home TV usage, but the basic system architecture explaining a geosynchronous spacecraft has not changed during this period.

With the launch of the first Iridium spacecraft in 1997 and 1998, a significant new architecture has been introduced into the field of satellite communications. These systems are based

\footnotetext{
Manuscript received October 5, 1998; revised June 11, 1999. This work was supported in part by Nortel Networks, Richardson, TX. The work of Y. Fang was supported in part by the New Jersey Institute of Technology under Grant SBR421 980 and the New Jersey Center for Multimedia Research.

Y. Xie is with the Erik Jonsson School of Engineering and Computer Science, University of Texas at Dallas, Richardson, TX 75083 USA (e-mail: tonyx @utdallas.edu).

Y. Fang is with the Department of Electrical and Computer Engineering, New Jersey Institute of Technology, Newark, NJ 08817 USA (e-mail: fang@oak.njit.edu).

Publisher Item Identifier S 0018-9545(00)03682-3.
}

upon the use of LEO and medium earth orbiting (MEO) systems. These LEO and MEO systems have several advantages over geosynchronous systems. The most significant advantages are: 1) the reduction in range provides a large decrease in path loss resulting in much small receiving antennas and 2) the reduction in range provides a significant reduction in propagation delay making voice conversation more pleasing to the user and increasing the throughput of most data communication protocols. These systems can and will serve mobile and portable users with small near omniantennas. However, the use of the small antennas as well as the motion of the transmitter and the receiver introduces the possibility of multipath and path blockage into the link budget of these satellite systems. Moreover, the propagation channel will be time varying due to different shadowing and scattering phenomena, so traditional channel models may not work well.

This paper is concerned with the statistical modeling of the propagation characteristics of LEO and MEO systems. Since in these systems, satellites and mobile users are all allowed to move during communication sessions, the channel characteristics will be different from the geostationary systems (GEO's). Due to the movement of receivers or transmitters, the received signals may fluctuate very rapidly from time to time. This fluctuation results from the combining effects of random multipath signals and obstruction of the line-of-sight path, which induces various fading phenomena. The communication quality of service (QoS) parameters such as the word-error rate will be affected in great deal in such communication environment. For effective mobile satellite communications system design, we must quantitatively know the propagation characteristics such as signal fading due to reflection; shadowing from trees, buildings, utility poles, and terrain; Doppler effects due to movement of mobile terminals, mobile satellites, or the communication effects; and other effects such as the rainfall. Such characteristics can be studied by the statistical distribution of the received signal envelope or received power in mobile communication systems. This paper is devoted to the statistical characterization of such propagation channels.

Ossanna [2] was the first to attempt to give an reasonable statistical characterization on the received mobile-radio signals using a set of interfering waves for land mobile communication environment. Clarke [3] systematically studied the same issue and derived the now famous Rayleigh distribution for the received signal envelope in urban areas based on the assumption that the received signal phase is rectangularly distributed in $[-\pi, \pi]$ and that the received multipath signal amplitudes are equal. After introducing the concept of large-scale fading and small-scale fading, Suzuki [4] gave the Rayleigh-lognormal 
model that was well tested in built-up areas and showed that this model was more appropriate for fitting the experimental data. As for the problem of modeling frequency-nonselective multipath fading and shadowing in the land-mobile satellite communication system, Loo [5] proposed a model suitable for rural environments, in which it assumed that the received signal was affected by frequency-nonselective Rice fading with lognormal shadowing on the direct component, the resulting model gave a generalization of the Suzuki's model. Along a similar line, Corazza and Vatalaro [6] presented another Rice-lognormal distribution, in which the shadowing affects both direct and diffuse components. Both Loo's model and Corazza-Vatalaro's model assumed that in the small-scale fading the strong component was constant, so that the Rice distribution can be used to characterize the small-scale fading. Recently, Patzold et al. [7] proposed an extended Suzuki model in which the $I$ component and the $Q$ component of the small-scale fading are allowed to be mutually correlated, which is the case when the Doppler power spectral density is asymmetrical.

All models discussed above are suitable for cases when the receiving terminals are fixed or mobile in a uniform environment-we call the model suitable for this environment the single-state model. When the terminals travel in a large area of a nonuniform environment, the received signals may change abruptly at different power levels, therefore, the received signals are more appropriately characterized by the what-so-called multistate model. Lutz et al. [8] introduced a two-state model, in which it was assumed that the received signals can experience two states: the "good state," corresponding to the case when the signals only undergo shadowing, and the "bad state," corresponding to the case when the signals experience strong component attenuation and weak signal fading. The multistate Markov model based on the combination of lognormal signal attenuation and Rician fading was proposed by Wakana [9]. Akturan and Vogel [10] introduced a three-state model, in which the Rician fading is used to characterize the clear state and Loo's models with different parameters are to characterize the shadowing state and the block state. Fontan et al. [11] used Loo's model with three different sets of parameters to construct their three-state Markov model. Osborne et al. [12] introduced the Corazza-Vatalaro model as the basis of their multistate model. Many of the above models have been studied and used in maritime and aeronautical satellite communication systems [13]-[18].

We observe that Loo's model and Corazza-Vatalaro's model are the composite models deriving from the Rice distribution (short-term fading) and the lognormal distribution (long-term fading). It is accepted that lognormal distribution is a good model for the long-term fading, while the Rice distribution is good only for some fading environments. In this paper, we propose a new general statistical model for mobile satellite communication systems based on propagation scattering theory, which describes the received signal as product of two independent processes: large- and small-scale fading. We still use the lognormal distribution to describe the large-scale fading. As for the small-scale fading, we use a more general distribution than Rice distribution. The new distribution model is derived based on a more general assumption on the distributions of the received signal amplitude and phase, so it can be used to characterize the fading in all types of environment, especially for the cases where there exist specularly reflected or diffracted components in received signals associated with the combination of randomly distributed stronger components and the weak scattering components. We show that all the single-state models discussed above are the special cases of this general model. This general model can also be used as the basis of the multistate model. Numerical results show that the general propagation model provides better fit to experimental data than other models available in the literature.

This paper is organized as follows. In Section II, we derive the general statistical model based on the propagation scattering theory. The probability density functions (pdf's) of the received signal envelope and the received signal power are given. The relationships between this model and the available models are explored. In Section III, numerical results for experimental data fitting for both the single-state model and the multistate model in land-mobile and mobile satellite communications are presented, and comparisons with results available in the current literature are made. We conclude this paper in Section IV.

\section{General Statistical Modeling FOR MOBILE COMMUNICATIONS}

As we discussed in the previous section, the design of mobile satellite communications systems to provide the desired communication quality of service depends on a good propagation model. We concentrate on the modeling for small-scale fading phenomena. We observe that in many mobile communication systems, the small-scale fading results from the reflections and refractions from different paths in particular when the elevation angle is low for land mobile satellite communications systems or the maritime satellite systems. Rice distribution is used to model such small-scale fading, and the hidden assumption behind this model is that the $I$ component and the $Q$ component of the fading signal have the same variances and that there is a strong constant component in the received signal. It seems that when the receiving terminal has low elevation angle or when it is used in maritime satellite systems with low-gain antenna, the received signals are the superposition of many scattering components whose amplitudes are randomly distributed in a wide range and whose phases are distributed over $(-\infty,+\infty)$. More importantly, the amplitudes and phases of the scattering components may be correlatted (which is the case when the rough surface is of finite conductance) [19], in which case the scattering phenomena really has a significant effect on the received signal. In this section, we propose a new general model based on the scattering theory of electromagnetic waves, which can to some extent capture such phenomena.

\section{A. Distribution Model for the Received Signal Envelope}

Any transmitted signals will experience signal fluctuations. In general, the fluctuations are caused by the combination of multipath received signals that have different amplitudes and 


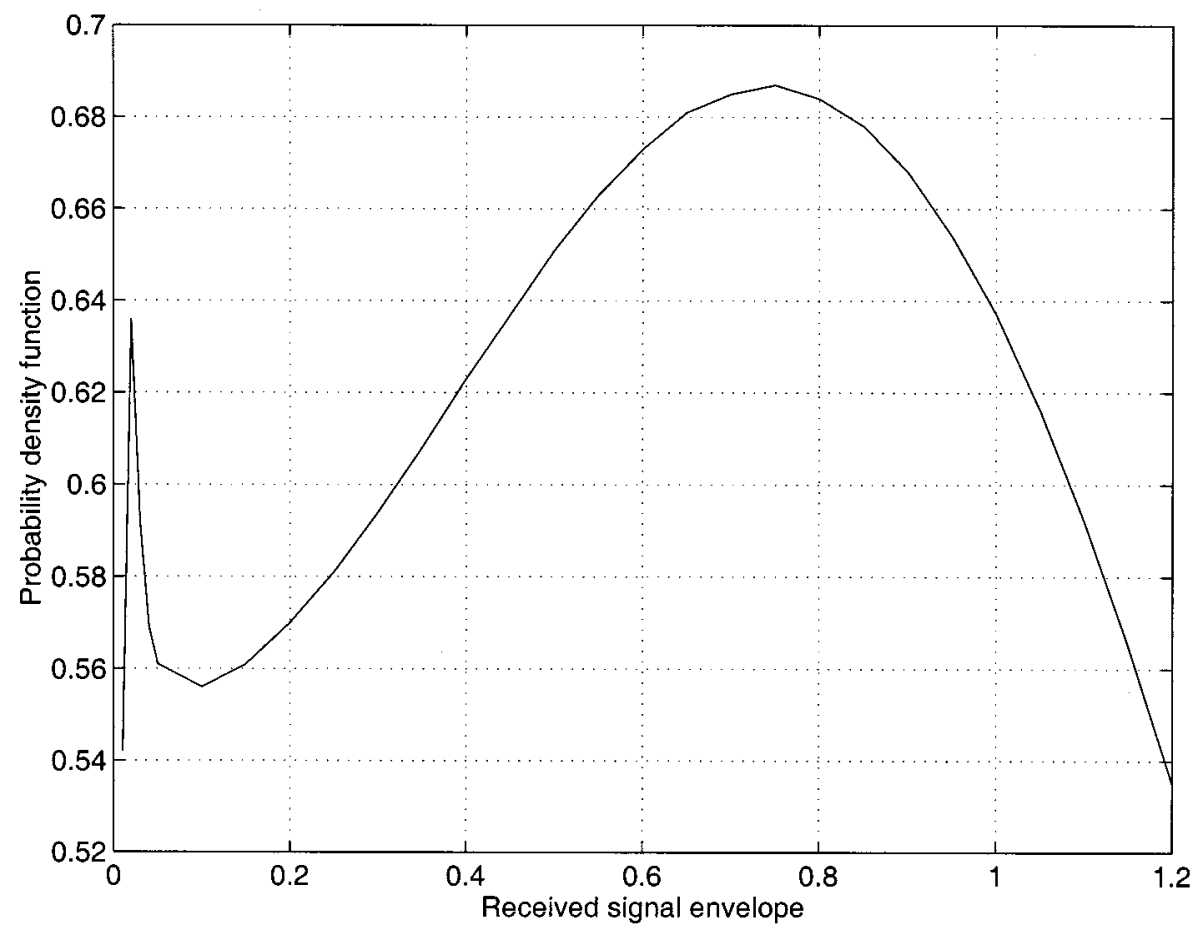

Fig. 1. Illustration of pdf of (9) with two peaks.

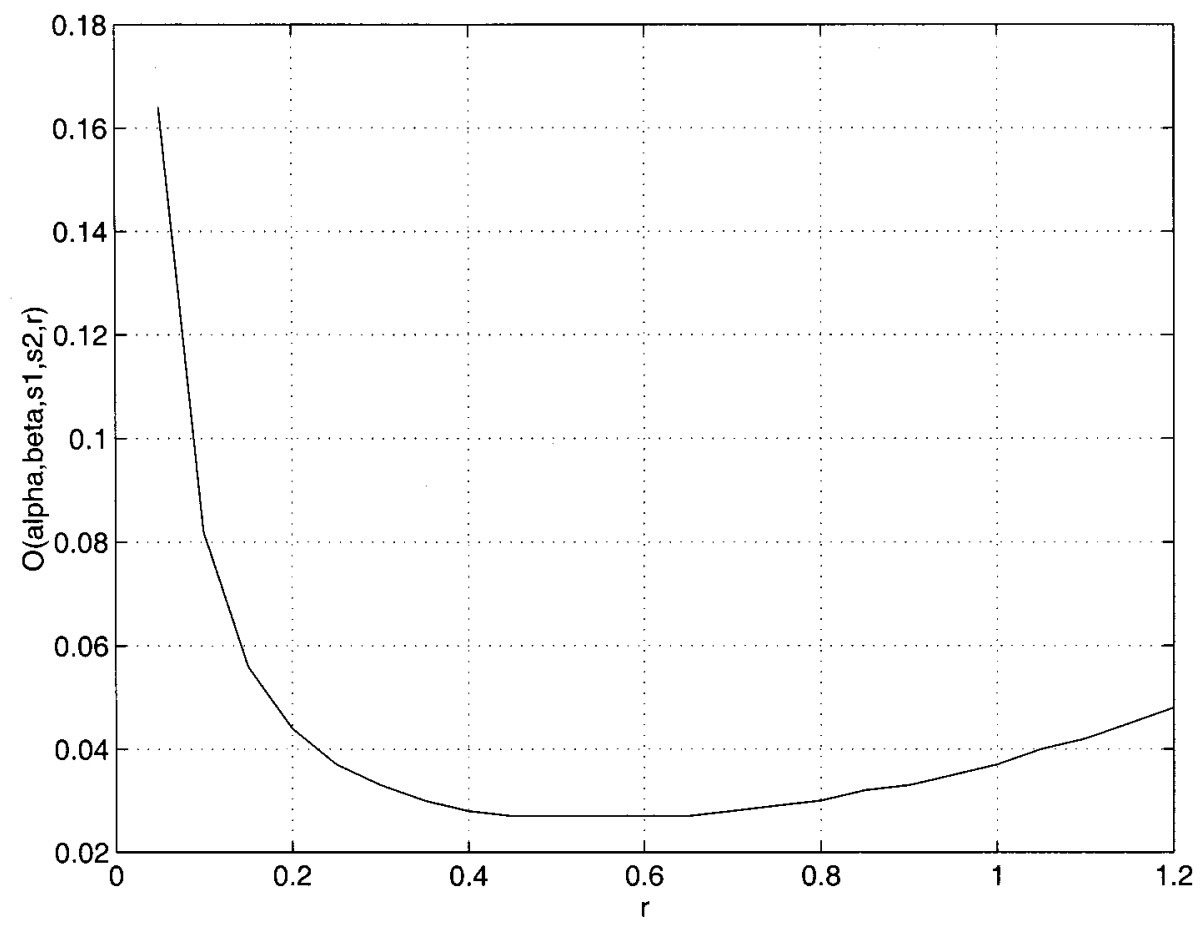

Fig. 2. The function $O\left(\alpha, \beta, s_{1}, s_{2}, r\right)$ with respect to $r$.

phases. Consider the received electrical field as the sum of such multipath signals [19]

$$
E=r e^{j \psi}=\sum_{i=1}^{n} A_{i} e^{j \phi_{i}}
$$

where $n$ is the number of multipath signals and the $A_{i}$ and $\phi_{i}$ are the amplitude and phase of the $i$ th path component, respectively. In general, $A_{i}$ and $\phi_{i}$ are time functions, and we omit the dependency of time for simplicity in this paper. Assume that $A_{i}$ is real and $\left(A_{i}, \phi_{i}\right)$ has the joint pdf $w_{i}(A, \phi)$. When all $A_{i}$ $(i=1,2, \cdots, n)$ are equal and the $\phi_{i}$ are uniformly distributed between $-\pi$ and $\pi$, then the resulting distribution when $n$ is infinite will lead to the Rayleigh distribution; when one component is significantly stronger than others, the resulting distribution is Rician. However, in many situations, different path signal will experience different attenuation and different phase shift, the 


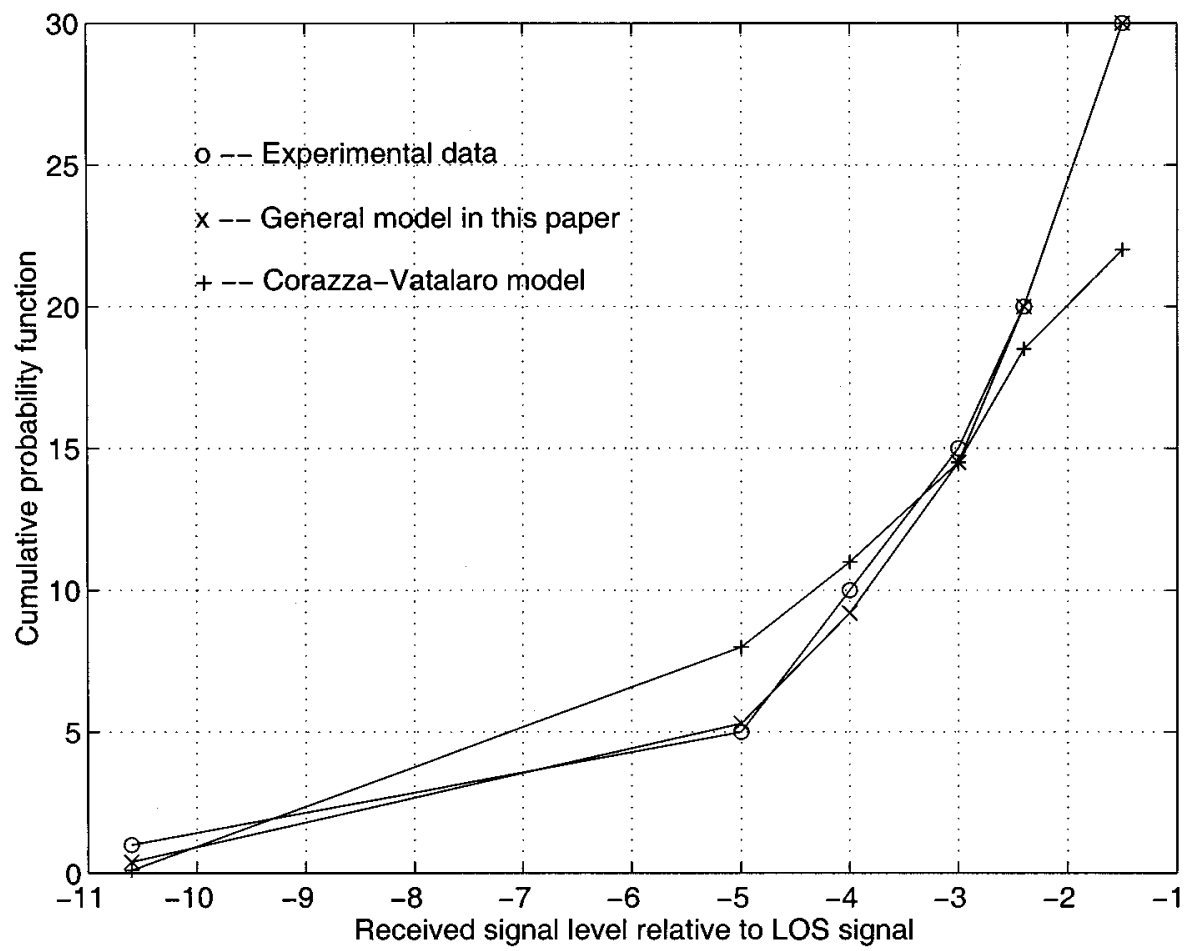

Fig. 3. Cumulative distribution function of the general model for $19^{\circ}$ elevation angle in open area in land-mobile satellite communication system compared with Corazza-Vatalaro's model.

asymmetrical Doppler spectrum observation [7] showed the correlated characteristics of $A_{i}$ and $\phi_{i}$, thus, it is better to study the general situation.

From (1), we obtain

$$
\begin{aligned}
& \operatorname{Re}(E)=x=r \cos (\psi)=\sum_{i=1}^{n} A_{i} \cos \left(\phi_{i}\right) \\
& \operatorname{Im}(E)=y=r \sin (\psi)=\sum_{i=1}^{n} A_{i} \sin \left(\phi_{i}\right)
\end{aligned}
$$

where Re and Im denote the real and imaginary parts of a complex quantity. From the above, we obtain

$$
\begin{aligned}
\alpha & =\langle x\rangle=\sum_{i=1}^{n} \int_{-\infty}^{\infty} \int_{0}^{\infty} w_{i}(A, \phi) A \cos (\phi) d A d \phi \\
\beta & =\langle y\rangle=\sum_{i=1}^{n} \int_{-\infty}^{\infty} \int_{0}^{\infty} w_{i}(A, \phi) A \sin (\phi) d A d \phi \\
s_{1} & =D\{x\}=<x^{2}>-\alpha^{2} \\
& =\sum_{i=1}^{n} \int_{-\infty}^{\infty} \int_{0}^{\infty} w_{i}(A, \phi) A^{2} \cos ^{2}(\phi) d A d \phi-\alpha^{2} \\
s_{2} & =D\{y\}=\left\langle y^{2}\right\rangle-\beta^{2} \\
& =\sum_{i=1}^{n} \int_{-\infty}^{\infty} \int_{0}^{\infty} w_{i}(A, \phi) A^{2} \sin ^{2}(\phi) d A d \phi-\beta^{2}
\end{aligned}
$$

where \langle\rangle denotes the expectation and $D$ denotes the variance. We observe [19] that if the phase and amplitude for each ray component are mutually independent, under certain symmetry
TABLE I

FORMULAS AND PARAMETERS

\begin{tabular}{l|l|l|l|l|l|l|l}
\hline & Formula & $\alpha$ & $\beta$ & $s_{1}$ & $s_{2}$ & $\mu$ & $\sigma$ \\
\hline Fig.1 & Eq.(9) & 0.0 & 0.8 & 0.01 & 0.6 & & \\
\hline Fig.2 & Eq. (10) & 0.0 & 0.8 & 0.01 & 0.6 & & \\
\hline Fig.3 & Eq. (13) & 0.52 & 0.66 & 0.20 & 0.25 & 0.14 & 0.70 \\
\hline Fig.4 & Eq. (13) & 0.56 & 0.795 & 0.20 & 0.39 & 0.0 & 0.30 \\
\hline Fig.5 & $0.81^{*}$ Eq.(18) & 0.0 & 1.04 & 0.01 & 0.12 & 0.0 & $\rightarrow 0$ \\
& $+0.19^{*}$ Eq.(18) & 0.0 & 0.0 & 0.2 & 0.99 & -6.1 & 1.8 \\
\hline Fig.6 & $0.85^{*}$ Eq.(13) & 0.65 & 0.70 & 0.1 & 0.1 & 0.0 & 0.2 \\
& $+0.15^{*}$ Eq.(13) & 0.11 & 0.48 & 0.1 & 0.232 & 0.0 & 0.1 \\
\hline
\end{tabular}

condition of the pdf we may have $\alpha=\beta=0$ and $s_{1}=s_{2}$ while $\alpha \neq \beta$ and $s_{1} \neq s_{2}$ if the amplitude and phase of each ray component are correlated, which is the case when the $I$ component and the $Q$ component are correlated.

From (2) and (3), we obtain that the received signal envelope is given by

$$
r=\left(x^{2}+y^{2}\right)^{1 / 2}
$$

From the central limit theorem, for infinite $n$, we know that $x$ and $y$ are Gaussian distributed with means $\alpha$ and $\beta$ and with variances $s_{1}$ and $s_{2}$, respectively. Thus, following the procedure similar to the ones in [19], we obtain the pdf of signal envelope $r$

$$
\begin{aligned}
p_{r}(r)= & \frac{r}{\sqrt{s_{1} s_{2}}} \exp \left(-\frac{s_{1} r^{2}+s_{2} \alpha^{2}+s_{1} \beta^{2}}{2 s_{1} s_{2}}\right) \\
& \times O\left(\alpha, \beta, s_{1}, s_{2}, r\right)
\end{aligned}
$$

where we have (10), given at the bottom of the next page.

Assume the receiving terminal is stationary or moving in a uniform environment and the link path between the satellite and 


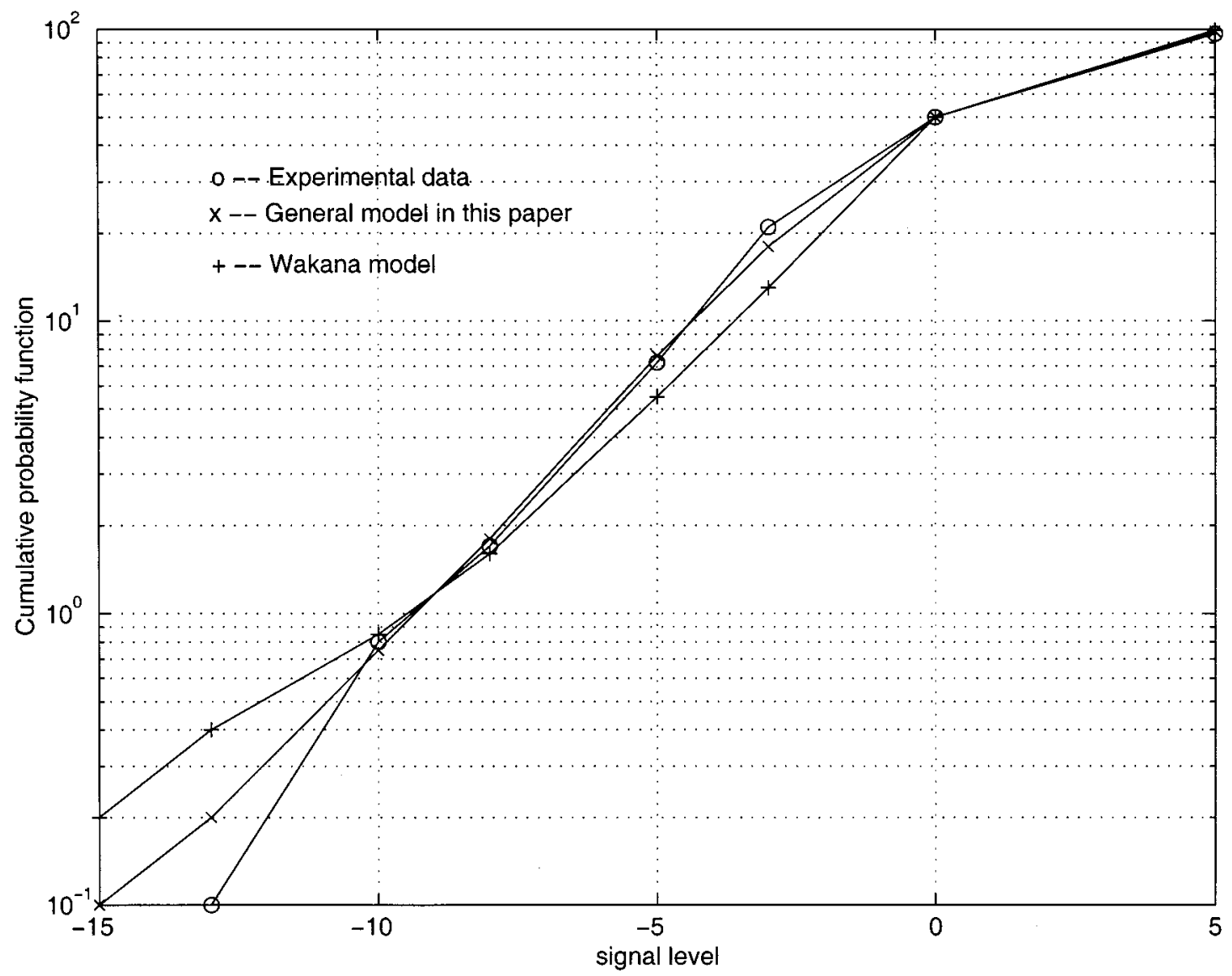

Fig. 4. Cumulative distribution function of the general model for $3^{\circ}$ elevation angle in maritime satellite communication system compared with Wakana's model.

the receiver is blocked by the obstructs such as buildings, trees, hills, and other types and scattered by environments. Using a similar argument in [6], we conclude that the received signal from satellite can be treated as a product of two independent processes

$$
R(t)=L(t) \cdot S(t)
$$

where $R(t)$ is the received signal, $L(t)$ is the large-scale fading that characterizes the signal attenuation over large transmitter-receiver separation distance, and $S(t)$ is the small-scale fading that characterizes the rapid fluctuations of the received signal over a short time duration.

Suppose that the small-scale fading can be characterized by the distribution given in (9) and assume that the large-scale fading is lognormal distributed with pdf

$$
p_{l}(l)=\frac{1}{\sqrt{2 \pi} \sigma l} \exp \left[-\frac{1}{2}\left(\frac{\ln l-\mu}{\sigma}\right)^{2}\right]
$$

where $\mu$ and $\sigma^{2}$ are the mean and variance of the associated Gaussian variable, respectively. From (11), we obtain the general pdf of received signal envelope in the mobile communication systems

$$
p(r)=\int_{0}^{\infty} \frac{1}{l} p_{r}\left(\frac{r}{l}\right) p_{l}(l) d l .
$$

\section{B. Probability Density Function of Received Signal Power}

The relationship between pdf of received signal envelope and that of received signal power is (from $w=r^{2}$ )

$$
p_{p}(w)=\frac{1}{2 \sqrt{w}} p_{r}(\sqrt{w})
$$

where $w$ denotes the received signal power. Thus, the smallscale pdf for the received signal power corresponding to (9) is given

$$
\begin{aligned}
p_{p}(w)= & \frac{1}{2 \sqrt{s_{1} s_{2}}} \exp \left(-\frac{s_{2} w+s_{2} \alpha^{2}+s_{1} \beta^{2}}{2 s_{1} s_{2}}\right) \\
& \times O_{p}\left(\alpha, \beta, s_{1}, s_{2}, w\right)
\end{aligned}
$$

$$
O\left(\alpha, \beta, s_{1}, s_{2}, r\right)=\frac{1}{2 \pi} \int_{0}^{2 \pi} \exp \left(\frac{2 s_{2} \alpha r \cos (\theta)+2 s_{1} \beta r \sin (\theta)+\left(s_{1}-s_{2}\right) r^{2} \cos ^{2}(\theta)}{2 s_{1} s_{2}}\right) d \theta
$$




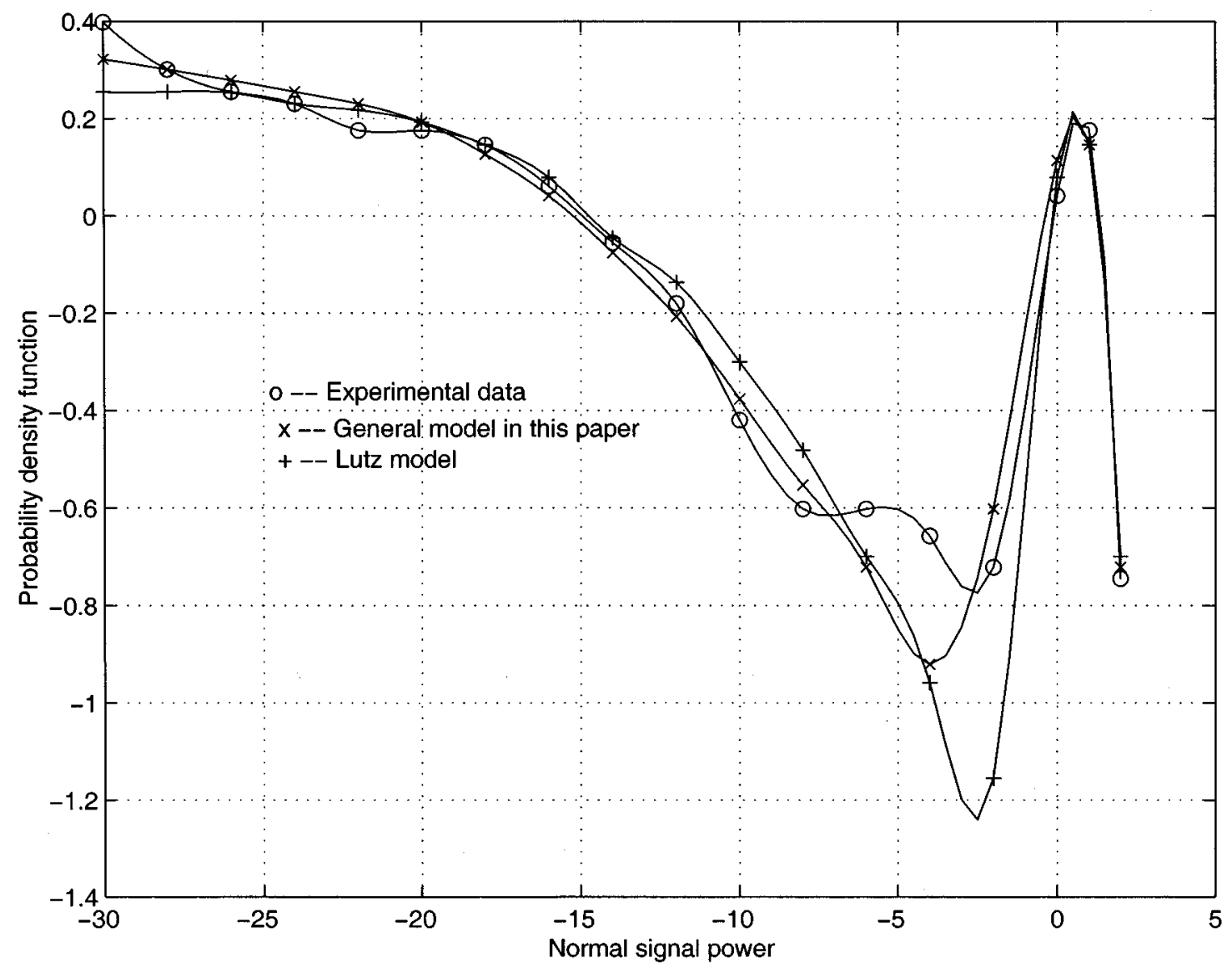

Fig. 5. Comparison of the pdf of multistate model as the linear combinations of (18) with results in [8].

where we have (16), given at the bottom of the page. If the large-scale fading is lognormally distributed with pdf [corresponding to (12)]

$$
p_{l_{0}}\left(l_{0}\right)=\frac{1}{\sqrt{2 \pi} \sigma l_{0}} \exp \left[-\frac{1}{2}\left(\frac{\ln l_{0}-\mu}{\sigma}\right)^{2}\right]
$$

where $\mu$ and $\sigma^{2}$ are the mean and variance of the associated Gaussian random variable, respectively, then the pdf of the received power corresponding to (13) is given by

$$
p(w)=\int_{0}^{\infty} \frac{1}{l_{0}} p_{p}\left(\frac{w}{l_{0}}\right) p_{l_{0}}\left(l_{0}\right) d l_{0}
$$

\section{Properties of the General Statistical Model}

In this section, we explore the relationship between the general model we have derived in the last section and the previously known models. We observe that the proposed models (13) and (18) contains all one-state models we discussed in the Introduction.
- When $\sigma=0, S(t)=1$, and $s_{1}=s_{2}$, the large-scale fading effect vanishes and (13) reduces to the Rice distribution. Furthermore, when $\alpha=\beta=0$, it reduces to the Rayleigh distribution.

- When $s_{1}=s_{2}$ and $\alpha=\beta=0$, the function $O\left(\alpha, \beta, s_{1}, s_{2}, r\right)$ in (9) is equal to one and then (13) reduces to the Suzuki model.

- When $s_{1}=s_{2}$ and $\alpha^{2}=\beta^{2}=\left(1-2 s_{1}^{2}\right) / 2$, the function $O\left(\alpha, \beta, s_{1}, s_{2}, r\right)$ becomes the modified Bessel function of the first kind of order zero and then (13) reduces to the Corazza-Vatalaro model.

- When $s_{1}=s_{2}$ and $\alpha=\beta=0$, (18) reduces to Rayleighlognormal distribution that was used to describe the bad state in [8].

As a final remark, we notice that in many traditional modeling approaches, we neglected many factors such as the correlation between the $I$ component and $Q$ component. In mobile satellite environments, propagation channels are usually time varying and scattering may be dominant (such as in the marine satellite links with low elevation angles), so the general model we present in this paper may capture those neglected factors.

$$
O_{p}\left(\alpha, \beta, s_{1}, s_{2}, w\right)=\frac{1}{2 \pi} \int_{0}^{2 \pi} \exp \left(\frac{2 s_{2} \alpha \sqrt{w} \cos (\theta)+2 s_{1} \beta \sqrt{w} \sin (\theta)+\left(s_{1}-s_{2}\right) w \cos ^{2}(\theta)}{2 s_{1} s_{2}}\right) d \theta
$$




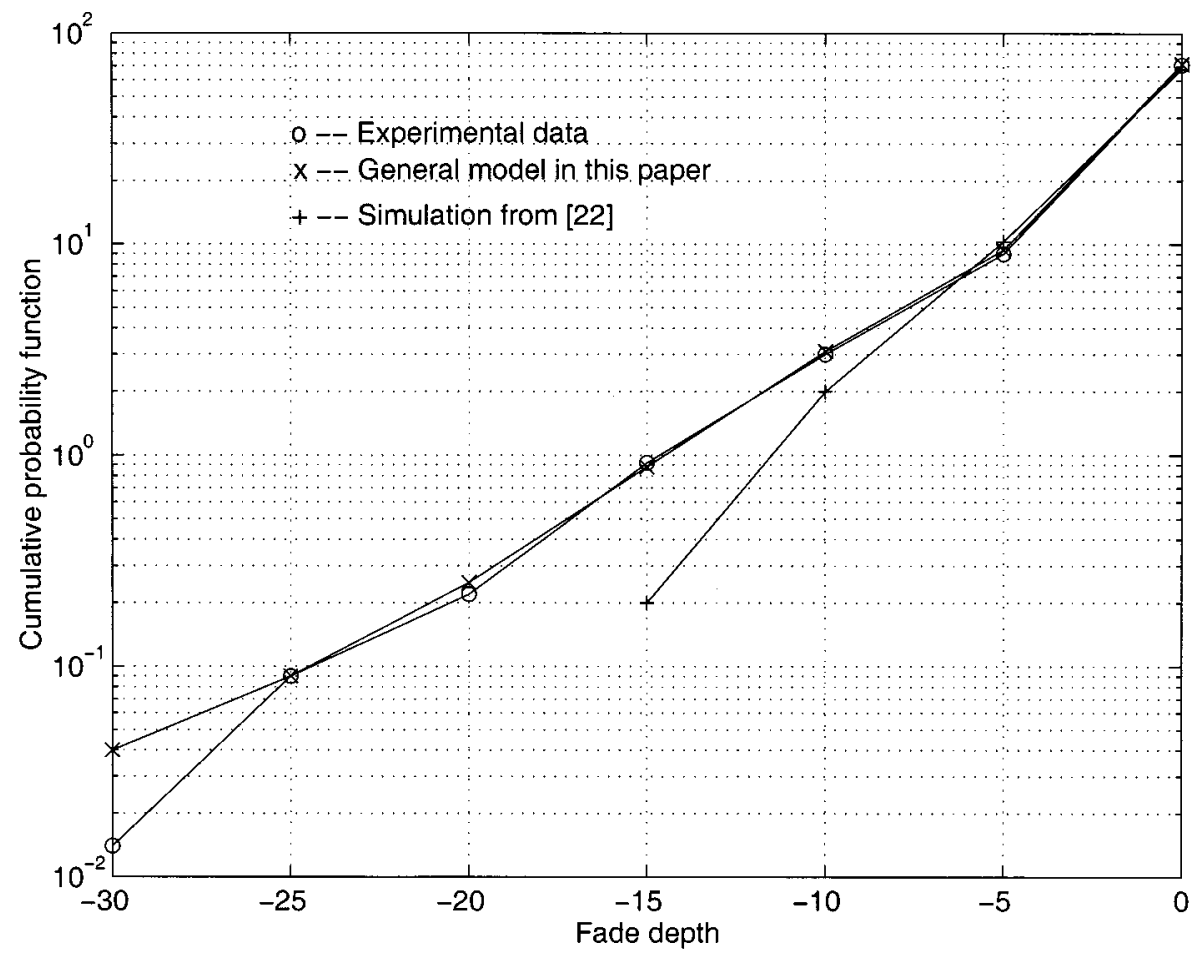

Fig. 6. Comparison of the cumulative probability function of multistate model as the linear combinations of (13) with the Markov transition simulation results in [14].

More importantly, the model we present here can be regarded as a general mathematical model, which contains more degrees of freedom to fit field data as we will show next.

\section{Applications of the General Statistical Model}

Since the general model we proposed is based on more realistic assumptions, we expect that our model could provide better fit for the experimental data, hence, it can be used in more general situations. In this section, we study this issue and present numerical comparisons with other known models to show the superiority of our new model.

Single-State Model: The single-state model for fading phenomena [22] is used to characterize the cases when receiving terminals travel in a terrestrial cellular network, or move in an uniform region in the geostationary/nongeostationary satellite communication systems, where the propagation channels do not have abrupt changes. The distribution of the received signal envelope can be described by (13) and the distribution of the received signal power can be described by (18). The large-scale term in these equations characterizes the slow variation in the received signal due to the variation of the terrestrial site. The rapid fluctuations in the received signal are caused by multipath effects in this site and are described by the small-scale term that is superimposed on the large-scale fading, which means all the components arriving at the terminal are influenced by the large-scale fading. Since we use more general assumption on the distribution for amplitude and phase of the received signal, rather than the assumption of the equal amplitude and rectangularly phase distribution between $-\pi$ and $\pi$ [2], [3], the function $O\left(\alpha, \beta, s_{1}, s_{2}, r\right)$ is introduced in (10). We see that in (9) there are four parameters which can be selected for data fitting, especially due to the introduction of function $O\left(\alpha, \beta, s_{1}, s_{2}, r\right)$, and the new model has much more freedom to choose than the Rice or the Rayleigh distribution.

For example, Fig. 1 shows the pdf of the general model, and we observe that there appear two peaks in the pdf that do not exist in Rice or Rayleigh pdf. This is possible for the general model because the function $O\left(\alpha, \beta, s_{1}, s_{2}, r\right)$ may decrease with respect to $r$ as shown in Fig. 2, while for the Rice or Rayleigh distribution the zero-order modified Bessel function of the first type always increases with respect to $r$.

From the derivation of the general model, we observe that the general model is suitable for any propagation environments, especially for the ones where there exist specularly reflected or diffracted components which are stronger than the random scattering signals. Fig. 3 shows the comparison of the cumulative distributions between the general model and the Corazza-Vatalaro's model, the parameters used in the general model are given in Table I. The parameters in Table I are obtained based on the curve fitting with the aid of a trial-and-error procedure for the fine tuning. We observe that because in open area there always exist random specularly reflected signals for low elevation angle in nongeostationary system, the general model indeed provides a better fit to the experimental data.

Fig. 4 shows the application of the general model (13) in the maritime satellite communication systems (corresponding formula and parameters are list in Table I). The sea surface is rough and moving, so the received signal are the combination of direct component and the scattering components caused by sea surface at low elevation angle. The roughness factor of the sea surface had been given and the Rician fading was proposed to model the 
received signal envelope [13]-[16], [23]. In general, the scattering components caused by the rough surface should be randomly distributed with more general distribution [19], hence, (13) is more appropriate for this environment, which is confirmed from Fig. 4 that the general model provides a better fit to the experimental data than Wakana's model.

Multistate Model: When the receiving terminal travels in a large area in geostationary or nongeostationary satellite communications systems, the received signals may change abruptly (say, change more than $10 \mathrm{~dB}$ ), which is the case when a mobile user travels from the open area to the urban area with densely populated building. It is not suitable to use the single-state model to characterize the slow variation corresponding to the large-scale fading. The multistate statistical model is more appropriate. The multistate model is the linear combination of several pdf's, each of which corresponds to the single-state model for a specific uniform environment [8]-[11]. Equations (13) or (18) can be used as the basis of the multistate model.

Fig. 5 shows the comparison between the result in [8] and that obtained from our general model (the corresponding formula and parameters are listed in Table I). In [8], the Rice distribution was used for "good state" and the Rayleigh-lognormal distribution for "bad state." In our model, we use two pdf's in the form of (18) with two sets of parameters. We can see that at the left end of the curves shown, our curve is closer to the experimental data, this is because in [8] the curve decreases exponentially, while in our model the function $O\left(\alpha, \beta, s_{1}, s_{2}, r\right)$ decreases faster with respect to $r$. Due to the similar reason, the deep "valley" of the curve obtained from [8] become flat in our model, which shows a better fit to the experimental data.

Fig. 6 presents the comparison of simulation result in [14] and that obtained from our general model. We can also observe the superiority of our general model to the experimental data fitting.

\section{CONCLUSION}

In this paper, we derive a general statistical model for mobile communication systems based on the propagation scattering theory. This model is shown to provide better characterization for different propagation environments compared with most previously known propagation channel models. We expect that this new channel modeling will play a significant role in providing better quality of service and more efficient system design for mobile communications systems, in particular, the mobile satellite communications systems.

\section{ACKNOWLEDGMENT}

The authors would like to express their gratitude to Dr. W. P. Osborne and the annonymous reviewers for their detailed comments which greatly improved the quality of this paper.

\section{REFERENCES}

[1] G. C. Hess, Handbook of Land-Mobile Radio System Coverage. Boston, MA: Artech, 1998

[2] J. F. Ossanna, "A model for mobile radio fading due to building reflections: Theoretical and experimental fading waveform power spectra," B.S.T.J., vol. 43, no. 6, pp. 2935-2971, Nov. 1964.
[3] R. H. Clarke, "A statistical theory of mobile-radio reception," B.S.T.J., vol. 47, pp. 957-1000, 1968.

[4] H. Suzuki, "A statistical model for urban radio propagation," IEEE Trans. Commun., vol. COM-25, no. 7, pp. 673-680, 1977.

[5] C. Loo, "A statistical model for a land mobile satellite link," IEEE Trans. Veh. Technol., vol. VT-34, no. 3, pp. 122-127, 1985.

[6] G. E. Corazza and F. Vatalaro, "A statistical model for land mobile satellite channels and its application to nongeostationary orbit system," IEEE Trans. Veh. Technol., vol. 43, no. 3, pp. 738-742, 1994.

[7] M. Patzold, U. Killat, and F. Laue, "An extended Suzuki model for land mobile satellite channels and its statistical properties," IEEE Trans. Veh. Technol., vol. 47, no. 2, pp. 617-630, 1998.

[8] E. Lutz, D. Cygan, M. Dippold, F. Dolainsky, and W. Papke, "The land mobile satellite communication channel-Recording, statistics, and channel model," IEEE Trans. Veh. Technol., vol. 40, no. 2, pp. 375-386, 1991.

[9] H. Wakana, "A propagation model for land-mobile-satellite communication," in Proc. IEEE Antennas and Propagation Society Symp., Canada, June 1991, pp. 1526-1529.

[10] R. Akturan and W. J. Vogel, "Path diversity for LEO satellite-PCS in the urban environment," IEEE Trans. Antennas Propagat., vol. 45, no. 7, pp. 1107-1116, 1997.

[11] F. P. Fontan, J. P. Gonzalez, M. J. S. Ferreiro, M. A. V. Castro, S. Buonomo, and J. P. Baptista, "Complex envelope three-state Markov model based simulator for the narrow-band LMS channel," Int. J. Sat. Commun., vol. 15, pp. 1-15, 1997.

[12] W. P. Osborne, Y. J. Xie, Y. G. Fang, and E. Roberts, "Channel modeling in LEO satellite mobile communication system," 2nd Tech. Rep. to Nortel, June 1998.

[13] Y. Karasawa and T. Shiokawa, "Characteristics of $L$-band multipath fading due to sea surface reflection," IEEE Trans. Antennas Propagat., vol. AP-32, pp. 618-623, June 1984.

[14] W. A. Sandrin and D. J. Fang, "Multipath fading characterization of L-band maritime mobile satellite links," COMSAT Tech. Rev., vol. 16, no. 2, pp. 319-338, 1986.

[15] S. Ohmori and S. Miura, "A fading reduction method for maritime satellite communications," IEEE Trans. Antennas Propagat., vol. AP-31, no. 1, pp. 184-187, 1983 .

[16] A. R. Miller, R. M. Brown, and E. Vegh, "New derivation for the rough surface reflection coefficient and for distribution of sea-wave elevations," Proc. Inst. Elect. Eng., pt. H, vol. 131, pp. 114-116, Apr. 1984.

[17] R. W. Sutton et al., "Satellite-aircraft multipath and ranging experiment results at $L$-band," IEEE Trans. Commun., vol. COM-21, pp. 639-647, May 1973.

[18] T. C. Jedrey, K. I. Dessouky, and N. Lay, "An aeronautical-mobile satellite experiment,” IEEE Trans. Veh. Technol., vol. 40, pp. 741-749, Nov. 1991.

[19] P. Beckmann and A. Spizzichino, The Scattering of Electromagnetic Waves from Rough Surfaces. Norwood, MA: Artech, 1987.

[20] A. Papoulis, Probabilities, Random Variables and Stochastic Processes, 3rd ed. New York: McGraw-Hill, 1991.

[21] J. G. Proakis, Digital Communications, 3rd ed. New York: McGrawHill, 1995.

[22] G. E. Corazza, A. Jahn, E. Lutz, and F. Vatalaro, "Channel characterization for mobile satellite communications," in Proc. 1st European Workshop on Mobile/Personal Satcoms (EMPS'94), London, U.K., 1995, pp. 225-250.

[23] S. Ohmori, H. Wakana, and S. Kawase, Mobile Satellite Communications. Boston, MA: Artech, 1998.

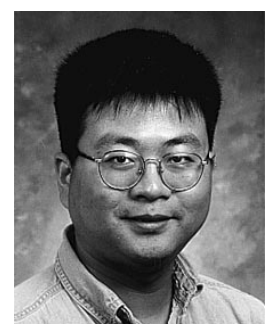

Yongjun Xie was born in Xian, China, in 1968. He received the B.S.E.E., M.S.E.E., and Ph.D. degrees from Xidian University, China, in 1990, 1993, and 1996 , respectively.

He had been an Associate Professor at Xidian University since June 1997. Currently, he is a Post-Doctoral Research Fellow at the University of Texas at Dallas, Richardson, TX. His research areas include microwave telecommunication theory and engineering, electromagnetic theory, and microwave technology. He has conducted several projects and has published more than 20 papers in these areas. 


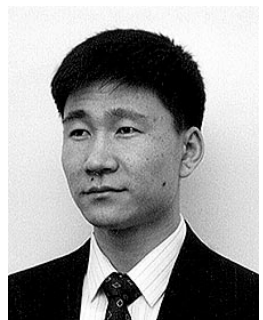

Yuguang Fang (S'96-M'98-SM'99) received the B.S. and M.S. degrees in mathematics from Qufu Normal University, Qufu, Shandong, China, in 1984 and 1987, respectively, the Ph.D. degree in systems and control engineering from Case Western Reserve University, Cleveland, OH, in 1994, and another $\mathrm{Ph} . \mathrm{D}$. degree in electrical and computer engineering from Boston University, Boston, MA, in May 1997.

From 1987 to 1988 , he held research and teaching positions at both the Department of Mathematics and the Institute of Automation at Qufu Normal University. From September 1989 to December 1993, he was a Teaching and a Research Assistant in the Department of Systems, Control and Industrial Engineering, Case Western Reserve University, where he was a Research Associate from January to May 1994. He held a Post-Doctoral position in the Department of Electrical Engineering, Boston University, from June 1994 to August 1995. From September 1995 to May 1997, he was a Research Assistant in the Department of Electrical and Computer Engineering, Boston University. From June 1997 to July 1998, he was a Visiting Assistant Professor in the Department of Electrical Engineering, University of Texas at Dallas. Since July 1998, he has been an Assistant Professor in the Department of Electrical and Computer Engineering, New Jersey Institute of Technology, Newark, NJ. He has published more than 40 papers in professional journals and refereed conferences. He is an Editor of the Wireless Communication Series for the IEEE JOURNAL ON SELECTED AREAS IN COMMUNICATIONS. His research interests include wireless networks and mobile communications, PCS's, mobile satellite communications, stochastic and adaptive systems, hybrid systems in integrated communications and controls, robust stability and controls, nonlinear dynamical systems, and neural networks.

Dr. Fang is the Area Editor for the ACM Mobile Computing and Communications Review. He is the Program Vice Chair for the Seventh International Conference on Computer Communications and Networking (IC ${ }^{3} \mathrm{~N}^{\prime} 98$ ), a member of the Technical Program Committee for the International Conference on Computer Communications (INFOCOM'98 and INFOCOM'00), a member of the Technical Program Committee for the First International Workshop for Wireless and Mobile Multimedia (WOW-MoM'98), and a member of the Technical Program Committee for the first International Conference on Wireless Communication and Networking (WCNC'99). 